\title{
Modélisation sur vingt ans (1976-1995) de la production phytoplanctonique en baie de Seine
}

\author{
Jean-François GULLLAUD, Alain MENESGUEN \\ Institut français de recherche pour l'exploitation de la mer (Ifremer), Centre de Brest, BP 70, 29280 Plouzané, France
}

(Revisé le 13 mars 1998, accepté le 24 mars 1998)

\begin{abstract}
Modelling over twenty years (1976-1995) of the phytoplanktonic production in the Bay of Seine. This paper presents an ecological modelling of the Bay of Seine (Eastern Channel) over the last twenty years, chosen as a typical case of eutrophication in a river plume. In the physical sub-model, the Bay is divided into 42 boxes and water fluxes hetween them are calculated automatically using Ifremer's "Flise" software. A two-layer, vertical thermohaline model is then linked with the horizontal circulation scheme in order to take vertical stratification into account. The biological submodel deals with two chemical elements, nitrogen and silicon, and splits phytoplankton into diatoms and flagellates. Results from this ecological model point out the spatial concordance of highest phytoplanktonic concentrations with the river plume spreading in the bay. Contrary to diatoms, flagellate production appears to be mainly confined to the eastern bay, due to the vertical haline stratification in front of the river mouth. As far as the whole bay is concerned, the interannual fluctuations of diatom production are related to the level of spring insolation, whereas silicon inputs regulate diatom production in the river plume. The flagellate summer production in the plume is enhanced by high water temperature and high $\mathrm{N} / \mathrm{Si}$ ratios, which appear during dry years with low discharge regimes. Finally, interannual increase of flagellate production could be related to gradual increase of nitrogen loadings, contrasting with silicon loadings, which remained stable for twenty years. (C) Elsevier, Paris
\end{abstract}

\section{two-layer model / Bay of Seine / diatom / flagellate / eutrophication}

Résumé - Le phénomène d'eutrophisation dans un panache fluvial est présenté par une modélisation écologique sur 20 ans de la baie de Seine (Manche orientale). Dans le sous-modèle physique, la baie est divisée en 42 boîtes et les flux d'eau interboîtes sont calculés grâce au logiciel Elise ; un modèle bicouche thermohalin est couplé au schéma de circulation horizontale afin de prendre en compte les stratifications verticales de la colonne d'eau. Le sous-modèle biologique considère l'azote et le silicium, et partage le phytoplancton en diatomées et flagellés. Les résultats de ce modèle écologique mettent en évidence la concordance géographique entre les zones de concentrations maximales du phytoplancton et l'extension du panache de la Seine dans la baie. La production des flagellés, contrairement à celle des diatomées, est localisée essentiellement dans la partic oricntale de la baie, cn raison de la stratification haline des eaux. Les fluctuations interannuelles de la production des diatomées sur l'ensemble de la baie sont liées aux variations de l'insolation printanière, alors que dans le panache elles dépendent des apports terrestres en silicium. La production estivale des flagellés dans le panache est accrue par des températures élevées de l'eau et par de fortes valeurs du rapport N/Si durant les années à étiage sévère. La tendance pluriannuelle à l'augmentation de la production des flagellés est à rapprocher de l'évolution des apports depuis vingt ans, caractérisée par une croissance en azote, et une stabilité en silicium.

modèle bicouche / baie de Seine / diatomée / flagellé / eutrophisation 


\section{INTRODUCTION}

Les poussées de phytoplancton marin dans les panaches de grands fleuves sont un phénomène largement reconnu en zone côtière, et qui semble évoluer quantitativement et qualitativement, en fonction de l'augmentation des apports continentaux de nutriments [43]. Une forte production $\mu_{1}$ : anare est ainsi observée dans la baie de Chesapcake [11], au large du Mississippi [48] et du Rhin [36], aux débouchés du Pô [24], de l'Elbe, de la Weser et de I'Ems [37], et en mer Baltique [23].

En France, parmi les zones soumises à l'influence des grands fleuves côtiers (Seine, Loire, Gironde, Rhône), c'est en baie de Seine que l'on observe les phénomènes d'eutrophisation les plus marqués; les concentrations en chlorophylle $a$ au débouché de l'estuaire de la Seine sont en moyenne de $8,7 \mu \mathrm{g} \mathbf{L}^{-1}$ (avril à septembre) el less valeurs maximales annuelles s'élèvent à environ $50 \mu \mathrm{g} \mathrm{L}^{-1}$ [39]. Par ailleurs la baie de Seine est soumise à l'apparition d'eaux colorées et de phytoplancton toxique, due essentiellement aux développements annuels de diatomées puis de dinoflagellés. En 1983, Dinophysis a été détecté pour la première fois en quantité importante en baie de Seine et a donné lieu à 125 cas d'intoxications alimentaires. Depuis cette date, le suivi régulier de Dinophysis en baie de Seine, en particulier sur le site d'Antifer, montre que les concentrations peuvent atteindre un million de cellules par litre en été [27].

Afin d'étudier les relations entre les apports fluviatiles de nutriments et la production phytoplanctonique dans la baie, un premier modèle mathématique a été développé par Ménesguen et al. [31]. Ce travail a montré que les variations de la production primaire annuelle dépendaient plus des conditions hydrologiques et climatiques que de l'évolution à long terme des concentrations en nutriments dans les rivières ; il a aussi mis en évidence la nécessité d'introduire la stratification haline des eaux au débouché de l'estuaire pour reproduire plus correctement les poussécs phytoplanctoniques, notamment dans la partie orientale de la baie.

L'objectif de cette étude est d'améliorer la compréhension du phénomène de production primaire dans la baie de Seine, en liaison avec les apports de sels nutritifs par les principales rivières (Seine, Orne, Vire et Douve). Un modèle bicouche en boîtes a été développé el utilisé pour simuler la production phytoplanctonique sur une période de 20 ans (1976-1995) durant lacuelle les concentrations en sels nutritifs dans les rivières ont connu une évolution notable.

\section{STRUCTURE DU MODÈLE}

Pour simuler les variations saisonnières et pluriannuelles de la production phytoplanctonique dans la baie de Seine, des choix ont été faits concernant la structure générale du modèle, en particulier :

- seule l'advection à long terme est considérée, au travers de la circulation résiduelle de marée ; le mélange par la marée est pris en compte par l'intermédiaire des coefficients de dispersion,

- la simulation de la production phytoplanctonique s'appuie sur les cycles biogéochimiques de l'azote et du silicium, car ces nutriments limitent en premier la production primaire dans la baie [49].

Le modèle est dérivé du modèle à deux couches fixes utilisé pour la baie de Seine par Ménesguen et al. [31], ainsi que du modèle intégral thermohalin à deux couches d'épaisseurs variables développé pour l'ensemble de la Manche par Ménesguen et Hoch [32].

\subsection{Sous-modèle hydrodynamique}

Le principe retenu consiste à découper la zone d'intérêt en une mosaique de compartiments géographiques ou "boîtes " (figure I), entre lesquelles les mouvements horizontaux des masses d'eau sont représentés par des flux advectifs résiduels de marée (unidirectionnels), doublés de flux dispersifs bidirectionnels représentant le mélange turbulent créé par l'alternance des courants de marée.

Dans chaque compartiment géographique, la colonne d'eau est scindée verticalement en deux couches par une pycnocline d'épaisseur nulle, dont la profondeur est calculée en permanence grâce à un modèle intégral thermohalin adapté du modèle initial de Niiler et Kraus [34]. La mise en cuvre de ce type de modèle impose l'introduction dans chaque compartiment géographique de trois variables d'état physiques: la température de l'eau, sa salinité et l'épaisseur de la couche d'eau [32].

En vue de distinguer le devenir des variables biogéochimiques dans l'eau et dans le sédiment, une boîte sédimentaire a été ajoutée sous chaque colonne d'eau, echangeant avec la boîte d'eau de fond sus-jacente les variables dissoutes par diffusion et les variables particulaires par les processus antagonistes de dépôt et d'érosion, en fonction de la vitesse du courant et de l'agitation de surface créée par le vent. 


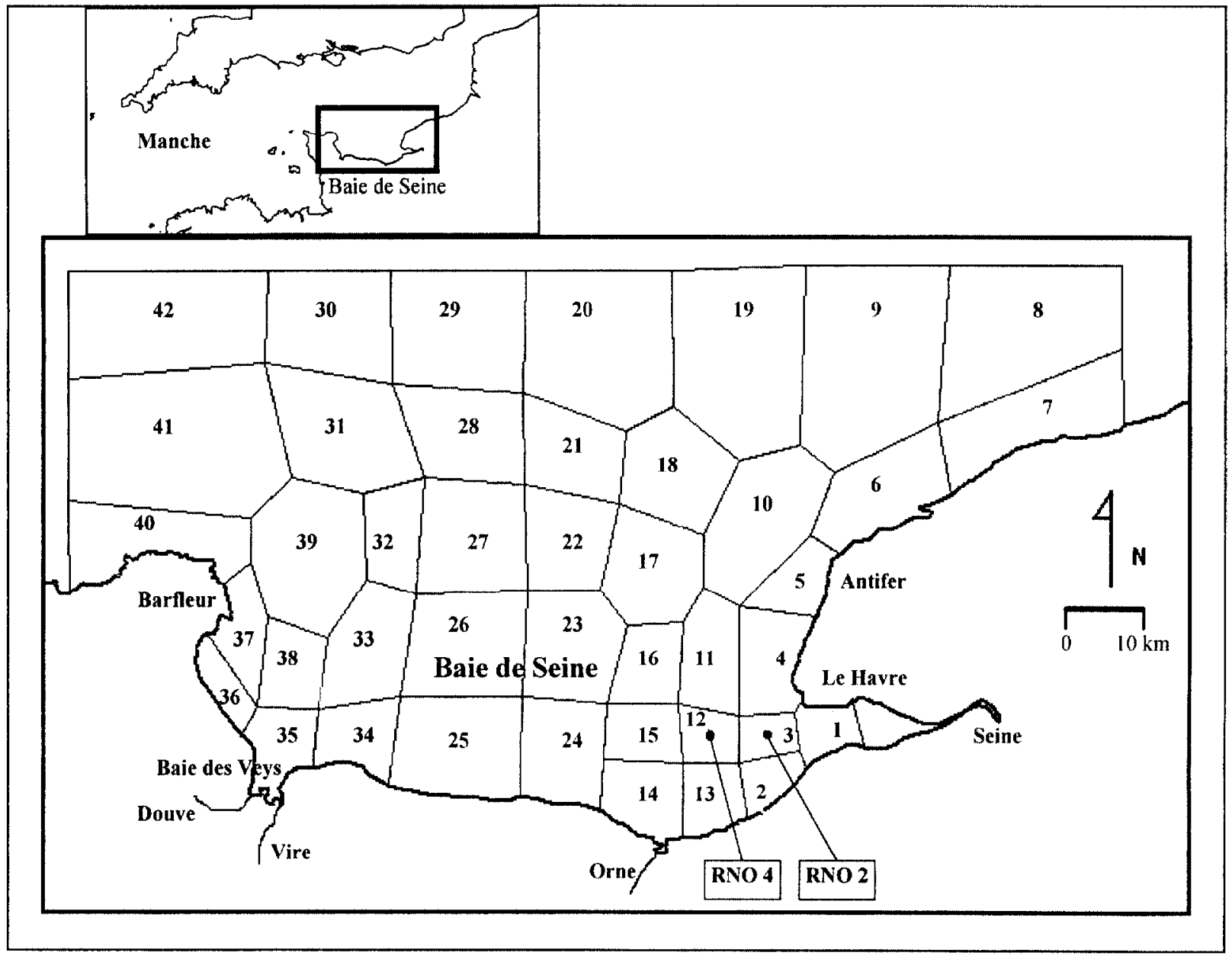

Figure 1. Position géographique de la baie de Seine, découpage en 42 bôtes du site et emplacement des stations RNO.

Figure 1. Map of the Bay of Seine, divided into 42 boxes, and location of RNO stations.

\subsection{Sous-modèle biogéochimique}

Représenté de façon schématique sur la figure 2, ce sousmodèle prend en compte les cycles de l'azote et du silicium de façon extrêmement simplifiée, par l'intermédiaire de six variables d'état, auxquelles on ajoute une septième variable nécessaire au calcul du coefficient d'extinction de la lumière : les matières minérales en suspension.

Les sept variables d'état du modèle biogéochimique sont :

$\mathrm{X}_{1}$ : azote inorganique dissous $\left(\mu \mathrm{mol} \mathrm{L}^{-1}\right)$

$\mathrm{X}_{2}$ : silice dissoute $\left(\mu \mathrm{mol} \mathrm{L}^{-1}\right)$

$\mathrm{X}_{3}$ : azote incorporé dans les diatomées $\left(\mu \mathrm{mol} \mathrm{L}^{-1}\right)$
$\mathrm{X}_{4}$ : azote incorporé dans les flagellés $\left(\mu \mathrm{mol} \mathrm{L} \mathrm{L}^{-1}\right)$

$\mathrm{X}_{5}$ : azote organique détritique $\left(\mu \mathrm{mol} \mathrm{L} \mathrm{L}^{-1}\right)$

$X_{6}$ : silice détritique biogène $\left(\mu \mathrm{mol} \mathrm{L} \mathrm{L}^{-1}\right)$

$\mathrm{X}_{7}$ : matières minérales en suspension $\left(\mathrm{mg} \mathrm{L}^{-1}\right)$

On considère que seules les diatomées incorporent de la silice, dans un rapport stoechiométrique $\left(\mathrm{r}_{\mathrm{Si} / \mathrm{N}}\right)$ supposé constant, ce qui évite d'ajouter une variable d'état pour la silice incorporée dans les diatomées. Comme dans le modèle élaboré pour la Manche entière par Ménesguen et Hoch [32], la croissance des deux catégories de phytoplancton n'est pas découplée de l'absorption des nutriments, ce qui aurait nécessité l'introduction d'un stockage interne aux algues (formulation à quota [13]). En effet, ce phénomène, dont la période caractéristique ne dépasse pas 


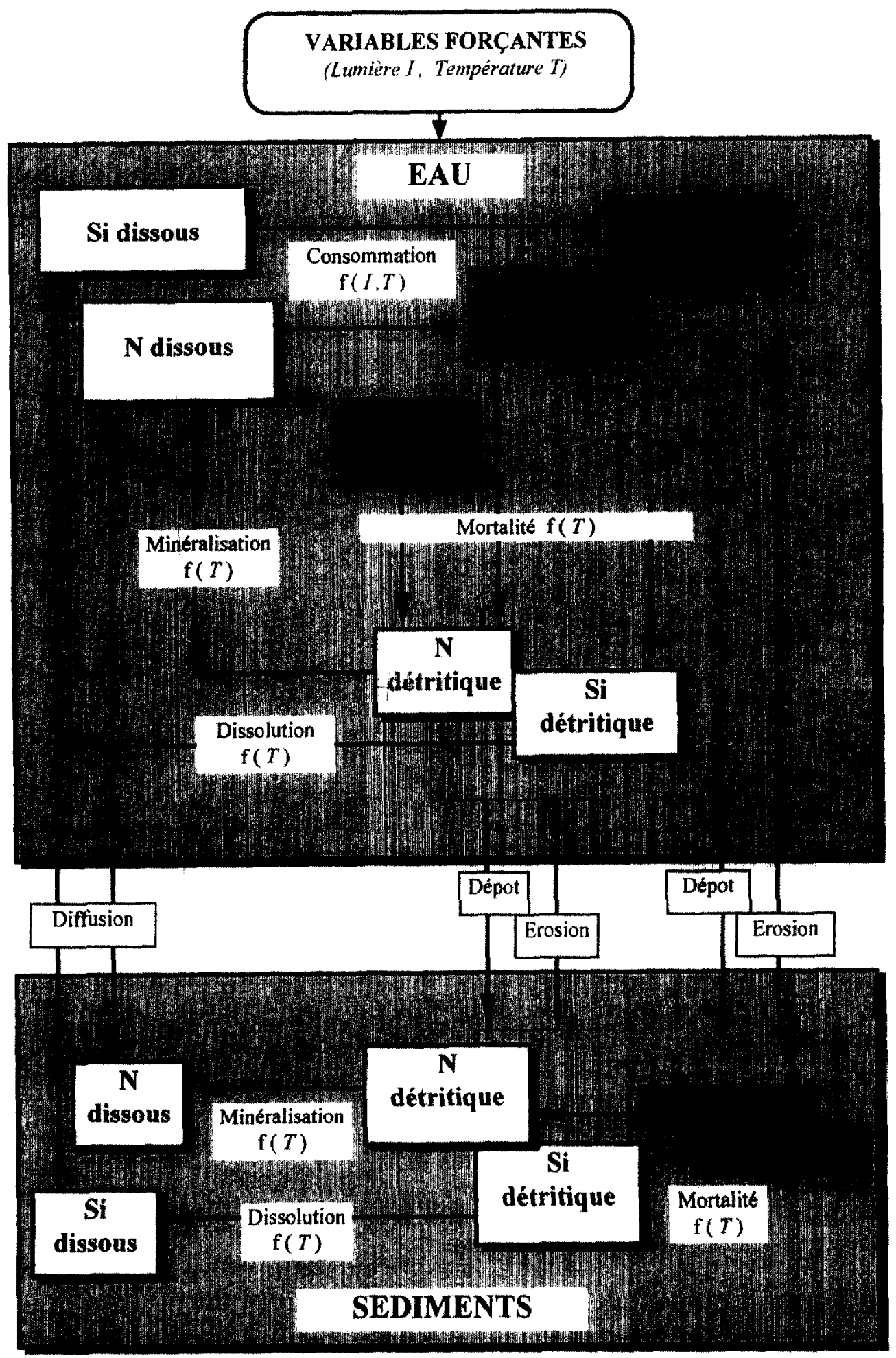

Figure 2. Schématisation du sous-modèle biogéochimique.

Figure 2. Flow-diagram of the biogeochemical sub-model. 
quelques jours, est négligeable dans les variations saisonnières et interannuelles qui sont la cible du modèle. La croissance des flagellés est donc limitée de façon michaëlienne par l'azote inorganique dissous dans l'eau, tandis que celle des diatomées est limitée, selon la loi de Liebig, par le minimum des effets michaëliens respectifs de l'azote et du silicium dissous. La lumière visible, considérée comme représentant la moitié de l'énergie solaire incidente dans le modèle thermique, agit sur la croissance des deux catégories de phytoplancton grâce à une fonction de Steele moyennée sur l'épaisseur de la couche d'eau. L'effet de la température est du type exponentiel (loi du $\mathrm{Q}_{10}=2$ ) sur la croissance et la mortalité phytoplanctoniques [15], comme d'ailleurs sur les autres processus sensibles à la température (reminéralisation de l'azote organique détritique, redissolution de la silice particulaire biogénique). La prédation par le zooplancton, qui n'est pas simulée, est intégrée dans le taux de mortalité du phytoplancton, en considérant un taux de mortalité des diatomées supérieur à celui des flagellés du fait d'une pression de broutage supérieure du zooplancton sur les diatomées [21]. Les diatomées, aịnsi que l'azote et la silice détritiques, sont considérés compne des variables particulaires dotées de vitesses de chute caractéristiques ; celle des diatomées est fonction de leur environnement nutritionnel [47], tandis que les flagellés, supposés capables de compenser leur chute passive par des mouvements verticaux, ne sont pas soumis à sédimentation dans le modèle. Le coefficient d'extinction de la lumière dépend des matières minérales en suspension selon une fonction puissance déterminée par Ménesguen et al. [31] à partir de nombreuses mesures en baie de Seine ; il dépend également des diatomées et des flagellés, selon la formule de Riley [40], les biomasses chlorophylliennes étant estimées à partir des biomasses azotées issues du modèle grâce au facteur de conversion d'une mole d'azote par gramme de chlorophylle $a$. Ce facteur a été déterminé en baie de Seine orientale par Aminot et al. [2] à l'aide des relations entre les déficits d'azote minéral (par rapport à la simple dilution) et les concentrations en pigments chlorophylliens durant le bloom à diatomées d'avril 1992 ; variant de 0,5 à $2 \mathrm{~mol} \mathrm{~g}^{-1}$, sa valeur tend vers $1 \mathrm{~mol} \mathrm{~g}^{-1}$ pour les fortes biomasses chlorophylliennes.

Dans l'établissement des équations différentielles régissant l'évolution locale des sept variables d'état dans une boîte d'eau (hors advection/dispersion et apports verticaux), les paramètres figurant au tableau I seront utilisés.

Les notations et expressions suivantes sont employées :

h : épaisseur de la couche d'eau (III)
$\mathrm{T}:$ temperature $\left({ }^{\circ} \mathrm{C}\right)$

fond : indicateur booléen égal à I si la boîte est en contact avec le sédiment $($ sinon $=0)$

Action de la température :

$f_{T}=\exp \left(k_{T} \cdot T\right)$

Action de la lumière :

$f_{L}=\iint\left(\frac{I_{z, t}}{I_{\text {sat }}}\right) \cdot \exp \left(1-\frac{I_{z, t}}{I_{\text {sat }}}\right) \cdot d z \cdot d t$

(formulation de Steele)

avec $I_{z, 1}$, intensité lumineuse visible à la profondeur $\mathrm{z}$ :

$I_{z, t}=I_{0, \tau} \cdot \exp \left(-k_{L} \cdot z\right)$

et $k_{\mathrm{L}}$ coefficient d'extinction lumineuse (formule de Riley modifiée) :

$k_{L}=k_{1} \cdot X_{7}^{k_{2}}+0,054 \cdot\left(X_{3}+X_{4}\right)^{\frac{2}{3}}+0,0088 \cdot\left(X_{3}+X_{4}\right)$

Action des nutriments sur la limitation de la croissance (formulation de Michaelis-Menten) :

- Azote : $f_{\text {Ndiat }}=\frac{X_{1}}{X_{1}+k_{\text {Ndiat }}}$ pour les diatomées ;

$$
f_{\text {Ndino }}=\frac{X_{1}}{X_{1}+k_{\text {Ndino }}} \text { pour les flagellés. }
$$

- Silicium : $f_{\text {Sidiat }}=\frac{X_{1}}{X_{1}+k_{\text {Sidiat }}}$ pour les diatomées

Vitesse de chute des diatomées:

$v_{\text {sdiat }}=v_{\text {sdiatmin }} \cdot$ nutstat $_{\text {diat }}+v_{\text {sdiatmax }} \cdot\left(1-\right.$ nutstat $\left._{\text {diat }}\right)$

avec nutstat $_{\text {diat }}$ représentant l'effet de la limitation nutritive sur la vitesse de chute des diatomées:

nutstat $_{\text {diat }}=\left[\min \left(f_{\text {Ndiat }}, f_{\text {Sidiat }}\right)\right]^{\frac{1}{5}}$

Les équations différenticlles régissant l'évolution de ces sept variables d'état dans une boîte d'eau sont les suivantes:

- pour l'azote inorganique dissous:

$$
\begin{aligned}
& \frac{d X_{1}}{d t}=r_{\min \mathrm{N}} \cdot f_{T} \cdot X_{5}-\mu_{\max \text { diat }} \cdot f_{T} \\
& \cdot \min \left(f_{\text {Sidiat }}, f_{\text {Ndiat }}, f_{\text {Ldiat }}\right) \cdot X_{3} \\
& -\mu_{\max \text { dino }} \cdot f_{T} \cdot \min \left(f_{\text {Ndino }}, f_{\text {Ldino }}\right) \cdot X_{4}
\end{aligned}
$$


Tableau 1. Valeurs des paramètres utilisés dans le sous-modèle biogéochimique

Table I. Parameter values used in the biogeochemical sub-model.

\begin{tabular}{|c|c|c|c|c|}
\hline Symbole & Définition & Unité & Valeur & Référence \\
\hline \multicolumn{5}{|l|}{ Diatomées } \\
\hline$\mu_{\text {maxdiat }}$ & taux de croissance maximal à $0^{\circ} \mathrm{C}$ & $j^{-1}$ & 0,45 & Chapelle, 1991 \\
\hline $\mathrm{l}_{\text {sardiat }}$ & intensité lumineuse optimale & $\mathrm{W} \mathrm{m}^{-2}$ & 70 & Mortain-Bertrand et al., 1988 \\
\hline $\mathrm{K}_{\mathrm{Ndiat}}$ & constante de demi-saturation pour l'azote minéral & $\mu \mathrm{mol} \mathrm{L} \mathrm{L}^{-1}$ & 2 & Eppley et al., 1969 \\
\hline $\mathrm{K}_{\text {Sidiat }}$ & constantc de demi-saturation pour la silice dissoute & $\mu \mathrm{mol} \mathrm{L} \mathrm{L}^{-1}$ & 1 & Paasche, 1973 \\
\hline$v_{\mathrm{sdiatmin}}$ & vitesse minimale de sédimentation & $\mathrm{m} \mathrm{j}^{-1}$ & 0,5 & Videau (comm. pers) \\
\hline$v_{\text {sdjatmax }}$ & vitesse maximale de sédimentation & $m j^{-1}$ & 1,2 & Videau (comm. pers) \\
\hline$m_{\text {diat }}$ & taux de mortalité à $0^{\circ} \mathrm{C}$ & $j^{-1}$ & 0,03 & Hoch, 1995 \\
\hline $\mathrm{r}_{\mathrm{Si} \mathrm{N}}$ & rapport $\mathrm{Si} / \mathrm{N}$ & $\mathrm{mol} \mathrm{mol}^{-1}$ & 0,5 & Harrison et al., 1977 \\
\hline \multicolumn{5}{|l|}{ Flagellés } \\
\hline$\mu_{\max \text { dino }}$ & taux de croissance maximal à $0^{\circ} \mathrm{C}$ & $j^{-1}$ & 0,20 & Calibration \\
\hline$I_{\text {satdino }}$ & intensité lumineuse optimale & $\mathrm{W} \mathrm{m} \mathrm{m}^{-2}$ & 180 & Andersen. 1985 \\
\hline $\mathrm{K}_{\text {Ndino }}$ & constante de demi-saturation pour l'azote minéral & $\mu \mathrm{mol} \mathrm{L} \mathrm{L}^{-1}$ & 3 & Le Pape, 1996 \\
\hline $\mathrm{mI}_{\mathrm{din} o}$ & taux de mortalité à $0^{\circ} \mathrm{C}$ & $j^{-1}$ & 0,02 & Hoch, 1995 \\
\hline \multicolumn{5}{|c|}{ Paramètres communs } \\
\hline $\mathrm{k}_{\mathrm{T}}$ & coefficient de l'effet thermique exponentiel & ${ }^{4} \mathrm{C}^{-1}$ & 0,07 & Eppley, 1972 \\
\hline \multicolumn{5}{|c|}{ Matière détritique } \\
\hline$r_{\min } \kappa^{-}$ & taux de reminéralisation de l'azote organique à $0^{\circ} \mathrm{C}$ & $j^{-1}$ & 0,05 & calibration \\
\hline$r_{\text {diss }}$ & taux de redissolution de la silice particulaire à $0^{\circ} \mathrm{C}$ & $\mathrm{j}^{-1}$ & 0,07 & calibration \\
\hline $\mathrm{v}_{\mathrm{SPOM}}$ & vitesse de chute de la matière organique détritique & $\mathrm{m} \mathrm{j}^{-1}$ & 1 & Hoch, 1995 \\
\hline$v_{\mathrm{sMES}}$ & vitesse de chute des matières en suspension minérales & $m j^{-1}$ & 0,3 & calibration \\
\hline $\mathrm{k}_{1}$ & $\begin{array}{l}\text { coefficient de la fonction puissance reliant les matières } \\
\text { en suspension et le coefficient d'extinction }\end{array}$ & $\mathrm{m}^{-1}\left(\mathrm{mg} \mathrm{L}^{-1}\right)^{-k 2}$ & 0,247 & Ménesguen et al., 1995 \\
\hline $\mathrm{k}_{2}$ & $\begin{array}{l}\text { exposant de la fonction puissance reliant les matières en } \\
\text { suspension et le coefticient d'extinction }\end{array}$ & sans dimension & 0,655 & Ménesguen et al.. 1995 \\
\hline$\tau_{\mathrm{cd}}$ & tension critique de dépôt de particules en suspension & $\mathrm{N} \mathrm{m} \mathrm{m}^{-2}$ & 0.2 & calibration \\
\hline$\tau_{\mathrm{ce}}$ & tension critique d'érosion du sédiment & $\mathrm{N} \mathrm{m}^{-2}$ & 0,3 & calibration \\
\hline resusp $p_{M E S}$ & $\begin{array}{l}\text { masse de particules minérales déposées remise en sus- } \\
\text { pension par unité de temps et unité de surface pour une } \\
\text { tension de frottement sur le fond égale à deux fois la } \\
\text { tension critique d'érosion }\end{array}$ & $\mathrm{kg} \cdot \mathrm{m}^{-2} \mathrm{~s}^{-1}$ & $10^{-5}$ & calibration \\
\hline
\end{tabular}

- pour la silice dissoute :

$$
\begin{aligned}
& \frac{d X_{2}}{d t}=r_{\text {diss }} \cdot f_{T} \cdot X_{6}-r_{\text {Si } / \mathrm{N}} \cdot \mu_{\text {max diat }} \cdot f_{T} \\
& \cdot \min \left(f_{\text {Sidiat }}, f_{\text {Ndiat }}, f_{\text {Ldiat }}\right) \cdot X_{3}
\end{aligned}
$$

- pour l'azote incorporé dans les diatomées:

$$
\begin{aligned}
& \frac{d X_{3}}{d t}=\left(\mu_{\text {max diat }} \cdot f_{T} \cdot \min \left(f_{\text {Sidiat }}, f_{\text {Ndiat }}, f_{\text {Ldiat }}\right)\right. \\
& -m_{\text {diat }} \cdot f_{T}-\frac{V_{\text {sdiat }}}{h} \\
& \left.\left(1+\text { fond } \cdot\left(\operatorname{Sup}\left[0,1-\tau / \tau_{c d}\right]-1\right)\right)\right) \cdot X_{3}
\end{aligned}
$$

- pour l'azote incorporé dans les flagellés:

$$
\frac{d X_{4}}{d t}=\left(\mu_{\max \text { dino }} \cdot f_{T} \cdot \min \left(f_{\text {Ndino }}, f_{\text {Ldino }}\right)\right.
$$

$$
\left.-m_{\mathrm{dino}} \cdot f_{T}\right) \cdot X_{4}
$$

- pour l'azote organique détritique:

$$
\begin{aligned}
& \frac{d X_{5}}{d t}=f_{T} \cdot\left(m_{\mathrm{diat}} \cdot X_{3}+m_{\mathrm{dino}} \cdot X_{4}\right) \\
& -\left(r_{\operatorname{minN}} \cdot f_{T}+\frac{v_{\mathrm{sPOM}}}{h}\right. \\
& \left.\cdot\left(1+\text { fond } \cdot\left(\operatorname{Sup}\left[0,1-\tau / \tau_{c d}\right]-1\right)\right)\right) \cdot X_{5}
\end{aligned}
$$


- pour la silice détritique biogénique:

$$
\begin{aligned}
& \frac{d X_{6}}{d t}=r_{\mathrm{Si} / \mathrm{N}} \cdot m_{\mathrm{diat}} \cdot f_{T} \cdot X_{3} \\
& -\left(r_{\mathrm{diss}} \cdot f_{T}+\frac{v_{\mathrm{sPOM}}}{h}\right. \\
& \left.\cdot\left(1+\text { fond } \cdot\left(\operatorname{Sup}\left[0,1-\tau / \tau_{c, d}\right]-1\right)\right)\right) X_{6}
\end{aligned}
$$

- pour les matières minérales en suspension:

$$
\frac{d X_{7}}{d t}=-\frac{v_{\text {sMES }}}{h} \cdot(1+\text { fond }
$$

$$
\left.\cdot\left(\operatorname{Sup}\left[0,1-\tau / \tau_{c, d}\right]-1\right)\right) \cdot X_{7}
$$

Dans la couche de sédiment, le modèle considère que le matériel détritique subit une reminéralisation dix fois plus lente que dans la colonne d'eau, alors que le phytoplancton sédimenté y meurt dix fois plus vite ; les nutriments issus de la reminéralisation diffusent ensuite vers la couche d'eau de fond. On utilise aussi l'hypothèse classique que la masse particulaire remise en suspension par unité de temps est proportionnelle à la tension $\tau$ de frottement sur le fond, pourvu que celle-ci soit supérieure à un seuil, appelé tension critique d'érosion $\tau_{c e}$. De même, le dépôt de particules ne se produit que si la tension $\tau$ de frottement sur le fond est inférieure à un seuil, appelé tension critique de dépôt $\tau_{\text {cud }}$. La tension $\tau$ de frottement sur le fond est créée par les actions combinées du courant de marée et des vagues; sa valeur est calculée selon les formules de Soulsby et al. [45].

La simplification des relations précédentes (Eqs. 1 à 7) p89ermet d'obtenir le jeu d'équations différentielles régissant l'évolution locale des sept variables d'état dans une boîte de sédiment (hors advection/dispersion et apports verticaux):

- pour l'azote inorganique dissous:

$\frac{d X_{1}}{d t}=\left(r_{\operatorname{minN}} / 10\right) \cdot f_{T} \cdot X_{5}$

- pour la silice dissoute:

$$
\frac{d X_{2}}{d t}=\left(r_{\mathrm{diss}} / 10\right) \cdot f_{T} \cdot X_{6}
$$

- pour l'azote incorporé dans les diatomées :

$$
\begin{aligned}
& \frac{d X_{3}}{d t}=-\left(10 \cdot m_{\mathrm{diat}}\right) \cdot f_{T} \cdot X_{3} \\
& - \text { resusp } \mathrm{MES} \cdot \operatorname{Sup}\left[0, \tau / \tau_{c e}-1\right] \\
& \cdot X_{3} /\left(C_{\mathrm{sed}} \cdot h_{\text {sed }}\right)
\end{aligned}
$$

- pour l'azote incorporé dans les flagellés :

$\frac{d X_{4}}{d t}=0$

de plus, dans le sédiment, $X_{4}=0$, car les flagellés ne sédimentent pas.

- pour l'azote organique détritique:

$$
\begin{aligned}
& \frac{d X_{5}}{d t}=\left(10 \cdot m_{\mathrm{diat}}\right) \cdot f_{T} \cdot X_{3}-\left(r_{\mathrm{minN}} / 10\right) \cdot f_{T} \cdot X_{5} \\
& -\operatorname{resusp} p_{\mathrm{MES}} \cdot \operatorname{Sup}\left[0, \tau / \tau_{c e}-1\right]
\end{aligned}
$$$$
\cdot X_{5} /\left(C_{\text {sed }} \cdot h_{\text {sed }}\right)
$$

- pour la silice détritique biogénique:

$$
\begin{aligned}
& \frac{d X_{6}}{d t}=r_{\mathrm{Si} / \mathrm{N}} \cdot\left(10 \cdot m_{\mathrm{diat}}\right) \cdot f_{T} \cdot X_{3} \\
& -\left(r_{\mathrm{diss}} / 10\right) \cdot f_{T} \cdot X_{6} \\
& -\operatorname{resusp}_{\mathrm{MES}} \cdot \operatorname{Sup}\left[0, \tau / \tau_{c e}-1\right] \\
& \cdot X_{6} /\left(C_{\mathrm{sed}} \cdot h_{\mathrm{sed}}\right)
\end{aligned}
$$

(Eq. 6bis)

- pour les matières minérales sédimentées:

$\frac{d X_{7}}{d t}=0 \quad$ et $\quad X_{7}=C_{\text {sed }}$

(Eq. 7 bis)

car on considère que les processus de dépôt et d'érosion, qui régissent les échanges particulaires entre le fond et la colonne d'eau sus-jacente, se font à densité de sédiment constante (sans tassement) et égale à $2,6 \times 10^{3} \mathrm{~kg} \mathrm{~m}^{-3}$; seule l'épaisseur $h_{\mathrm{sed}}$ de la couche sédimentaire varie selon l'intensité relative du dépôt et de l'érosion ; cette érosion obéit à l'équation différentielle suivante (le dépôt étant traité dans la boîte d'eau de fond) :

$\frac{d h_{\mathrm{sed}}}{d t}=-$ resusp $\mathrm{MES} \cdot \operatorname{Sup}\left[0, \tau / \tau_{c e}-1\right] / C_{\text {sed }}(\mathrm{Eq} .8 \mathrm{bis})$ 


\subsection{Conditions aux limites}

\subsubsection{Conditions au large}

La circulation résiduelle en Manche est telle que les entrées d'eau du large dans le modèle se font essentiellement au travers de sa frontière ouest ; les valeurs aux limites du modèle biologique ont été recherchées dans la zone située au large de Cherbourg. Des moyennes mensuelles ont été calculées pour la température, la salinité, les concentrations en azote minéral dissous et en silicate à partir des observations de Bentley et Maillard-Quisthoudt [7], Le Fèvre-Lehoërff et al. [26], Tappin [46], le Cnexo [10] et l'IOS [22]. L'azote des diatomées a été estimé à l'aide des teneurs en chlorophylle et d'un rapport azote/chlorophylle de $1 \mu \mathrm{mol} \mathrm{gg}^{-1}$ [2]. L'azote des flagellés varie de $0,0025 \mu \mathrm{mol} \mathrm{L^{-1 }}$ en hiver à $0,04 \mu \mathrm{mol} \mathrm{L}^{-1}$ en été [20]. Les concentrations en matières en suspension sont prises égales à $0,5 \mathrm{mg} \mathrm{L}^{-1}$ [2]. Les valeurs de silice détritique et d'azote détritique sont considérées comme nulles.

\subsubsection{Conditions fluviales}

Les débits, les températures et les concentrations en azote minéral et organique, en silicate et en matières en suspension dans les eaux de la Seine ont été fournis par la Cellule antipollution de la Seine (1976-1995); la station de mesures bimensuelles prise en compte est celle de Caudebec, située $40 \mathrm{~km}$ en amont de l'embouchure. La teneur en silice détritique a été estimée à partir des mesures de la biomasse pigmentaire du phytoplancton fluvial et d'un rapport silicium/pigments de $0,5 \mu \mathrm{mol} \mu \mathrm{g}^{-1}$ [2]. Pour les autres rivières bas-normandes (Orne, Vire et Douve) des données mensuelles sur les mêmes paramètres ont été mises à notre disposition par la Direction régionale de l'environnement et par l'Agence de l'eau Seine-Normandie. Pour le silicate, le petit nombre de mesures n'a permis de calculer, pour chaque rivière, qu'une évolution mensuelle des valeurs, considérée comme reproductible sur la période 1976-1995. Enfin, les concentrations en azote phytoplanctonique (diatomées et flagellés marins) sont considérées comme nulles dans les rivières.

L'évolution la plus remarquable de ces conditions aux limites fluviales concerne l'augmentation de $2,1 \%$ par an des concentrations en azote minéral dissous en Seine, entre 1976 et 1995 ; elles sont ainsi passées en moyenne annuelle de $340 \mu$ mol $\mathrm{L}^{-1}$ à $500 \mu \mathrm{mol} \mathrm{L} \mathrm{L}^{-1}$, en vingt ans

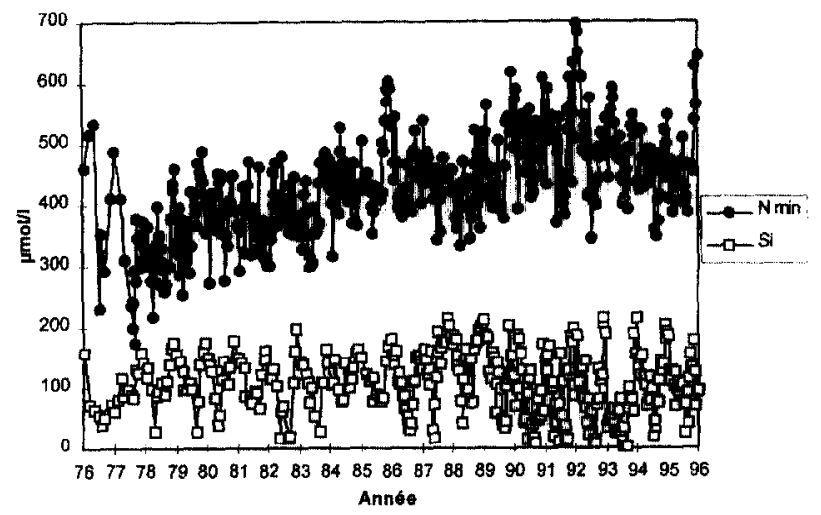

Figure 3. Variation des concentrations en azote minéral dissous et en silicate en Seine à Caudebec de 1976 à 1995.

Figure 3. Time-series of dissolved inorganic nitrogen and silicate in the River Seine (Caudebec, 1976-1995).
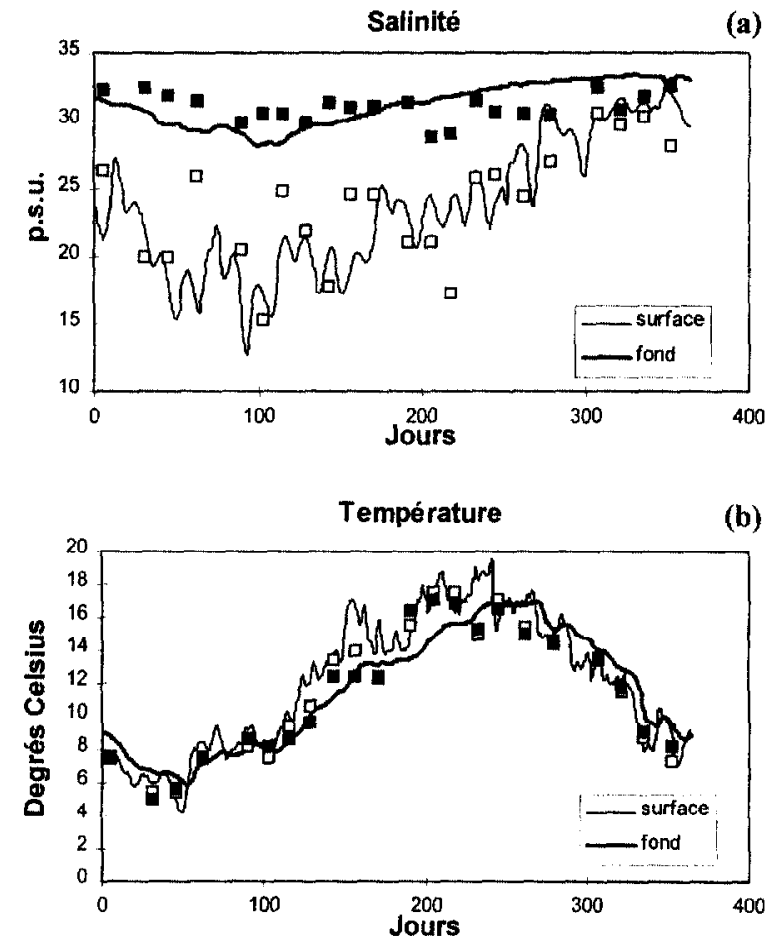

Figure 4. Comparaison des valeurs mesurées $(\square)$ et calculées ( - ) de la salinité (figure 4a) et de la température (figure 4b) à la station 2 du RNO en 1978.

Figure 4. Measured ( $\square$ ) and simulated (-) values of salinity (figure $4 \mathrm{a}$ ) and temperature (figure $4 \mathrm{~b}$ ) at RNO station No. 2 in 1978. 


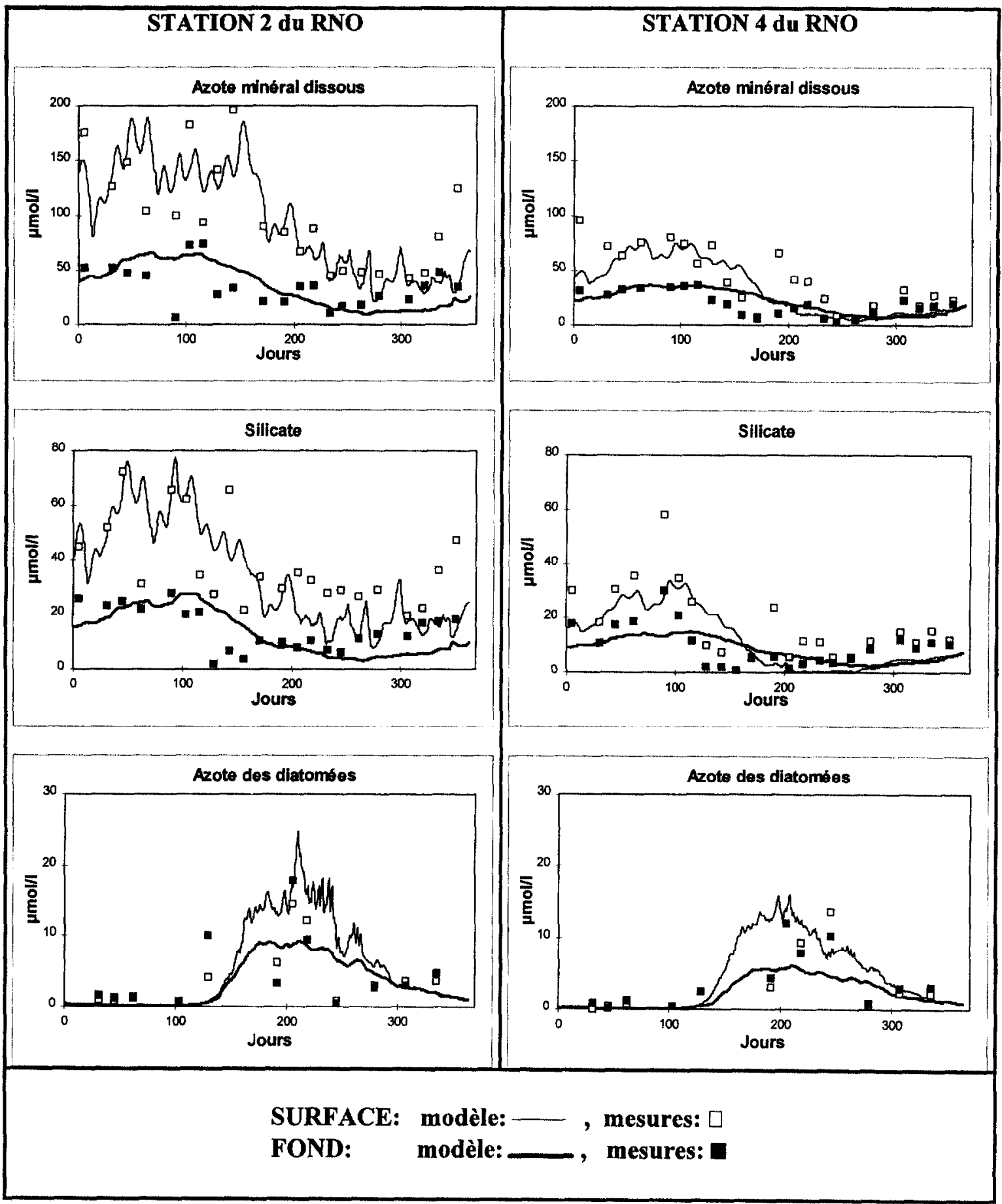

Figure 5. Comparaison des valeurs mesurées et calculées de l'azote minéral dissous, du silicate et de l'azote des diatomées, en surface et au fond, aux stations 2 et 4 du RNO en 1978.

Figure 5. Measured and simulated values of dissolved inorganic nitrogen, silicate and diatom nitrogen, in surface and bottom waters, at RNO stations No. 2 and No. 4 in 1978 . 
[31]. Les teneurs en silicium sont restées stables sur la même période (figure 3). Une évolution similaire de l'azote minéral dissous est observée sur les autres rivières bas-normandes.

\subsection{Variables forçantes}

Les variables forçantes utilisées dans le modèle sont la température et l'humidité relative de l'air, ainsi que la durée du jour, l'insolation, la nébulosité, la pression atmosphérique et la vitesse du vent. Les valeurs quotidiennes de ces variables à la station météorologique de la Hague (et de Cherbourg ou Caen pour certaines valeurs manquantes à la Hague) ont été introduites dans le modèle ; elles proviennent, pour la période 1976-1995. des services de Météo-France.

\section{RÉSULTATS}

\subsection{Calibration}

Le modèle a été calibré sur l'année 1978, première année pour laquelle on dispose de campagnes de mesures sur l'ensemble de la baie [2] et de données sur les stations du Réseau national d'observation de la qualité du milieu marin.

Une première calibration a été faite à l'aide des mesures réalisées à la station 2 du Réseau national d'observation de la qualité du milieu marin (RNO, 1978), qui se trouve dans l'embouchure, au sud du cap de la Hève. La figure 4 illustre à cette station la comparaison entre, d'une part, les mesures de salinité et de température en surface et au fond, et, d'autre part, les résultats du modèle après calibration, dans les boîtes de surface et de fond correspondantes. À l'exception d'une surestimation des valeurs de salinité au fond en fïn d'année, le modèle reproduit correctement le rôle des apports d'eau douce sur la stratification haline de la colonne d'eau. L'évolution des températures de surface est correctement simulée par le modèle ; en revanche les températures calculées au fond s'écartent plus nettement des valeurs mesurées, et engendrent des gradients thermiques verticaux plus importants que ceux qui sont réellement observés. Cette figure illustre enfin le rôle prépondérant de la salinité, par rapport à la température, sur la stratification verticale des eaux dans le panache. Dans le modèle cette stratification se traduit par une couche de surface dont l'épaisseur varie, dans le panache, entre 2 et $7 \mathrm{~m}$.
La figure 5 montre l'ajustement entre les valeurs calculées et les valeurs mesurées d'azote minéral dissous, de silicate, et d'azote des diatomées, en surface et au fond, aux stations 2 et 4 du RNO. En ce qui concerne les nutriments, l'ajustement est relativement correct à l'exception d'une régénération automnale plus tardive dans le modèle en particulier pour le silicate. Les valeurs calculées d'azote des diatomées, qui représentent la quasi-totalité de l'azote phytoplanctonique, ont été comparées aux mesures de chlorophylle $a$, à l'aide de l'équivalence déterminée par Aminot et al. [2] d'une micromole d'azote par microgramme de chlorophylle; l'évolution annuelle des biomasses phytoplanctoniques est globalement reproduite, avec des différences surface-fond plus importantes dans le modèle qu'en nature, témoignant d'une large répartition des diatomées sur la colonne d'eau.

La calibration du modèle a été faite par comparaison entre les concentrations en chlorophylle observées sur l'ensemble de la baie à une époque donnée, et la distribution géographique des valeurs calculées pour l'azote des diatomées à cette même époque. La figure 6 illustre cette comparaison entre valeurs calculées et valeurs observées en surface entre le 19 et le 23 mai 1978, avec une bonne correspondance géographique des valeurs maximales, qui se situent au large de l'embouchure. Cet éloignement, par rapport à l'estuaire, du maximum de biomasse phyloplanctonique est fréquemment observé au printemps en raison du rôle limitant de la turbidité des eaux [2]. En revanche le modèle donne des valeurs supérieures dans la partic ouest de la baic. La comparaison effectuée en septembre 1978 met en évidence, sur le modèle et en nature, un confinement des fortes biomasses phytoplanctoniques dans l'embouchure plus marqué qu'au printemps: mais là encore les valeurs calculées dans la partic ouest de la baie sont un peu plus élevées que les valeurs mesurées entre le 23 septembre et le 4 octobre 1978.

Enfin la calibration des paramètres intervenant dans le calcul de l'azote des flagellés a nécessité de se référer aux comptages cellulaires réalisés à Antifer tous les $15 \mathrm{j}$ depuis 1992. Les nombres de cellules de flagellés ont été convertis en azote, en estimant le contenu cellulaire à 4 pmol d'azote par cellule [20, 30].

La figure 7 montre que le modèle ne reproduit correctement que l'ordre de grandeur des concentrations en azote des flagellés en surface, mais ne donne pas les valeurs correctes des pics annuels observés. Le modèle donne des concentrations en azote des flagellés très faibles au fond, conformément aux observations en baie de Seine [29] qui 


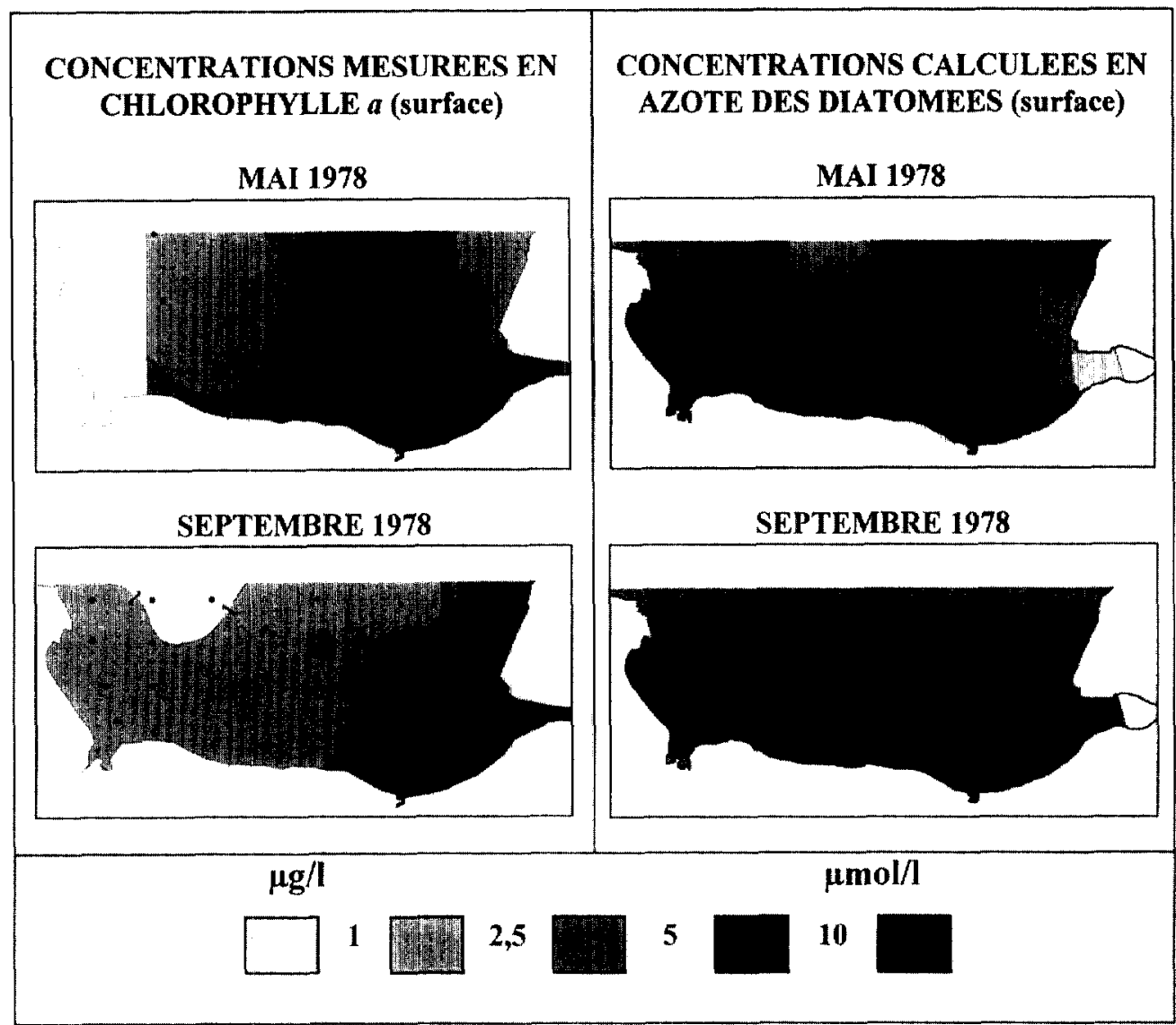

Figure 6. Concentrations mesurées en chlorophylle $a$ et concentrations calculées en azote des diatomées en surface dans la baie de Seine, en mai 1978 et en septembre 1978.

Figure 6. Measured chlorophyll $a$ concentrations and simulated diaton nitrogen concentrations in surface water of the bay, in May and September 1978.

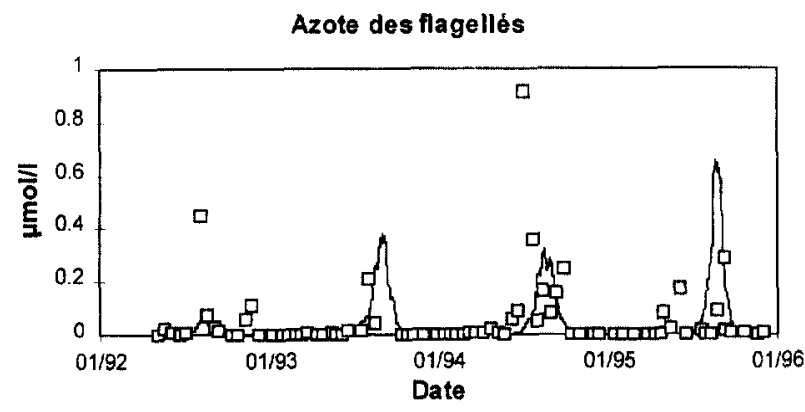

Figure 7. Concentrations observées ( $\square$ ) et calculées ( - ) en azote des flagellés en surface à Antifer de 1992 à 1995.

Figure 7. Measured $(\square)$ and simulated $(-)$ concentrations of flagellate nitrogen in surface water at Antifer (1992-1995). montrent qu'en période estivale plus de $90 \%$ des flagellés se trouvent dans les dix premiers mètres; elles confirment le rôle des stratifications halines sur la production des flagellés dans le panache; ces résultats peuvent être rapprochés des observations de Peperzak et al. [36] qui mettent en relation la stratification des eaux et la présence de Dinophysis acuminata dans le panache des eaux du Rhin en période estivale.

Les valeurs des paramètres biologiques résultant de cette calibration. ainsi que celles issues de la bibliographie, sont regroupées dans le tableau $I$.

\subsection{Validation}

Le modèle a été utilisé pour calculer, en surface et au fond, les concentrations en azote minéral, en silicate et en 
azote des diatomées dans la baie de Seine entre 1976 et 1984, période pour laquelle de nombreuses mesures de nutriments et de chlorophylle $a$ sont disponibles aux stations du Réseau national d'observation de la qualité du milieu marin [38]. Les mesures effectuées en surface et au fond à la station 2 , et les résultats du modèle dans les bô̂tes correspondantes, sont présentés sur la figure 8 . On observe un ajustement correct pour le silicate et pour l'azote minéral dissous, à l'exception de l'année 1977 et de l'hiver 1981-1982. Les valeurs calculées d'azote des diatomées montrent une correspondance relativement correcte avec les mesures de chlorophylle $a$, sans toutefois que l'ensemble des pics obscrvés en surface ct au fond soit parfaitement simulé.

Des coefficients de variation entre les valeurs calculées et les valeurs mesurées en surface et au fond ont été déterminés selon la formule proposée par Andersen et Nival [5] :

$C=\left[\sum_{i=1}^{n}\left(x_{c}-x_{o}\right)^{2}\right]^{1 / \omega} / n x_{m}$

où $x_{c}$ est la valeur calculée, $x_{o}$ est la valeur observée, $x_{m}$ est la moyenne des $x_{o}$, et $n$ le nombre de couples de valeurs

Les valeurs de $C$, très voisines en surface et au fond, sont respectivement 0,04 pour le silicate, 0,05 pour l'azote minéral dissous, et 0,08 pour l'azote des diatomées ; elles indiquent un meilleur ajustement pour le silicate et pour l'azote minéral dissous que pour l'azote des diatomées. Elles sont tout à fait comparables aux valeurs jugées satisfaisantes par Andersen et Nival [5] et par Aksnes et al. [3], lors de la simulation des mêmes variables en mésocosmes.

La validation du modèle a aussi consisté à comparer, durant l'année 1992, la répartition géographique des concentrations en chlorophylle mesurées en surface, et les valeurs calculées en azote des diatomées au même niveau (figure 9). Au mois de février les concentrations en phytoplancton sont faibles sauf devant l'Orne et dans la baie des Veys où elles présentent une légère augmentation. Au mois d'avril les concentrations mesurées s'élèvent, notamment dans la partie est de la baie et au large de l'estuaire ; une structure assez voisine est retrouvée par le modèle. Au mois de juin, les mesures et le modèle mettent en évidence de fortes biomasses au débouché immédiat de l'estuaire.
Enfin une validation qualitative a été tentée en comparant les valeurs annuelles maximales d'azote des flagellés, données par le modèle dans la zone du panache, et les concentrations maximales de Dinophysis sp., mesurées dans le port d'Antifer depuis 1983 [12, 25, 27]. Sans être parfaitement simultanés, ces pics de flagellés apparaissent généralement au mois d'août en nature et dans le modèle. La comparaison a été faite après conversion du nombre de cellules de Dinophysis sp. en azote phytoplanctonique ( 4 pmol par cellule). Tout en reconnaissant les limites de cette approche qui ignore les espèces autres que Dinophysis sp. dans l'estimation de la biomasse observée de flagellés, on observe une certaine cohérence entre l'évolution des valeurs maximales calculées, et la succession des maxima de Dinophysis sp. observés à Antifer, notamment entre 1983 et 1992 (figure 10).

\subsection{Exploitation du modèle}

Le modèle a d'abord été exploité pour déterminer la concentration moyenne en azote phytoplanctonique dans chaque boîte de surface, afin d'avoir une représentation globale sur vingt ans des zones les plus riches. Pour les diatomées il apparaît que le secteur adjacent à l'estuaire de la Seine et, dans une moindre mesure, la baie des Veys, présentent les plus fortes concentrations ; l'ensemble de la baie de Seine et la zone côtière orientale montrent une augmentation significative des valeurs par rapport aux eaux de la Manche ouest. En ce qui concerne l'azote des flagellés, les concentrations maximales sont localisées dans la partie est de la baie, avec un maximum près de la côte, entre l'estuaire de l'Orne et l'embouchure de la Seine (figure 11). Cette localisation, ainsi que l'absence de développement important de flagellés dans la partie ouest de la baie, sont confirmées par les observations de Le Grand [27].

$\mathrm{Si}$, au lieu de la biomasse, on considère la production totale d'azote phytoplanctonique sur l'ensemble du site couvert par le modèle, la production des diatomées $s$ 'effectue pour seulement $11 \%$ en moyenne dans la zone du panache de la Seine. En revanche ce même panache contribue en moyenne à $38 \%$ de la production totale des flagellés dans la zone modélisée ; ce pourcentage s'élève à plus de $50 \%$ durant les années de forte production de flagellés.

La production annuelle des diatomées (exprimée en tonnes d'azote), calculée sur l'ensemble de la baie est comprise entre 360000 t/an et 480000 t/an ; les fluctuations de cette production sont liées de façon significative 


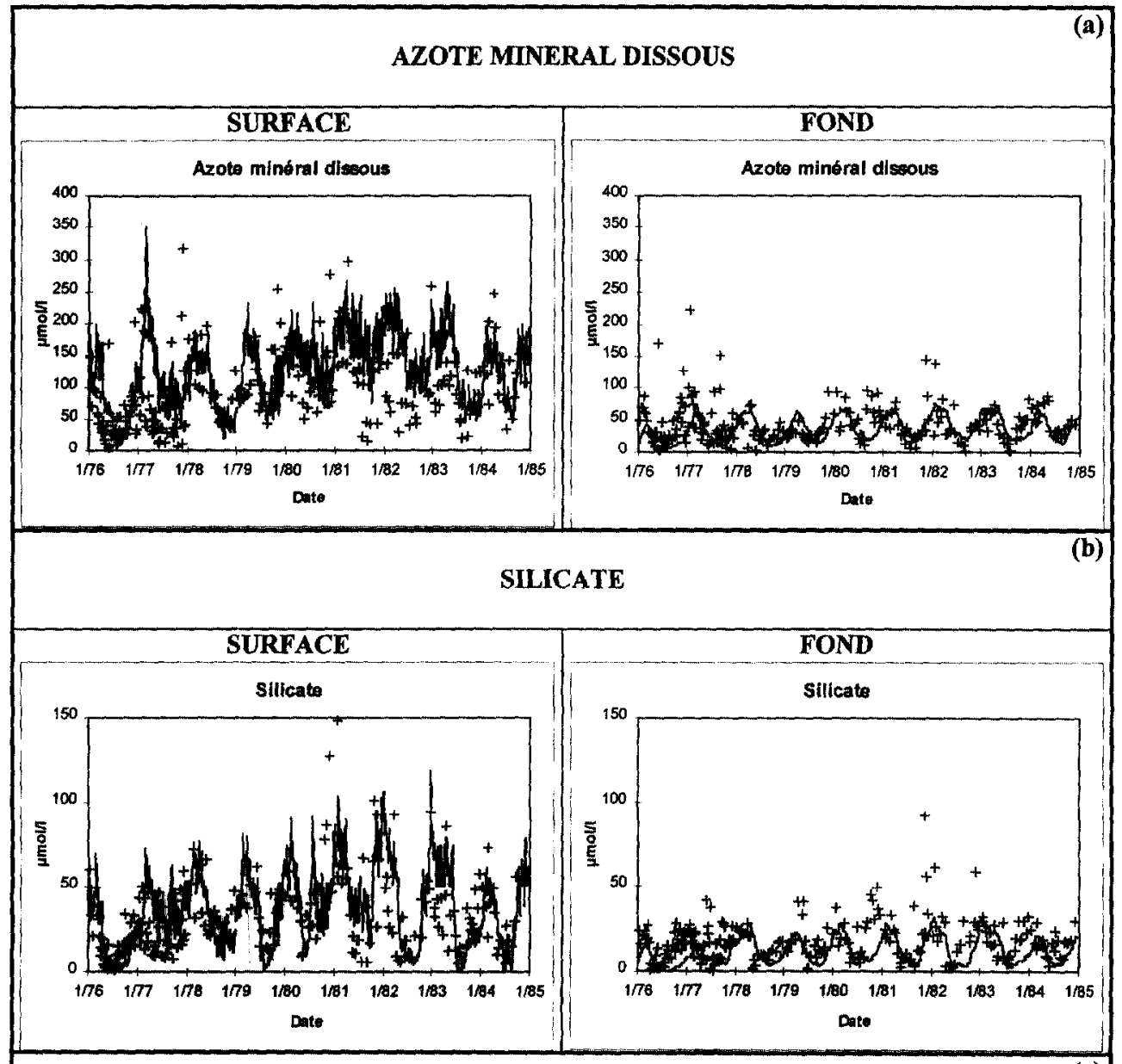

AZOTE DES DIATOMEES ET CHLOROPHYLLE a

(c)

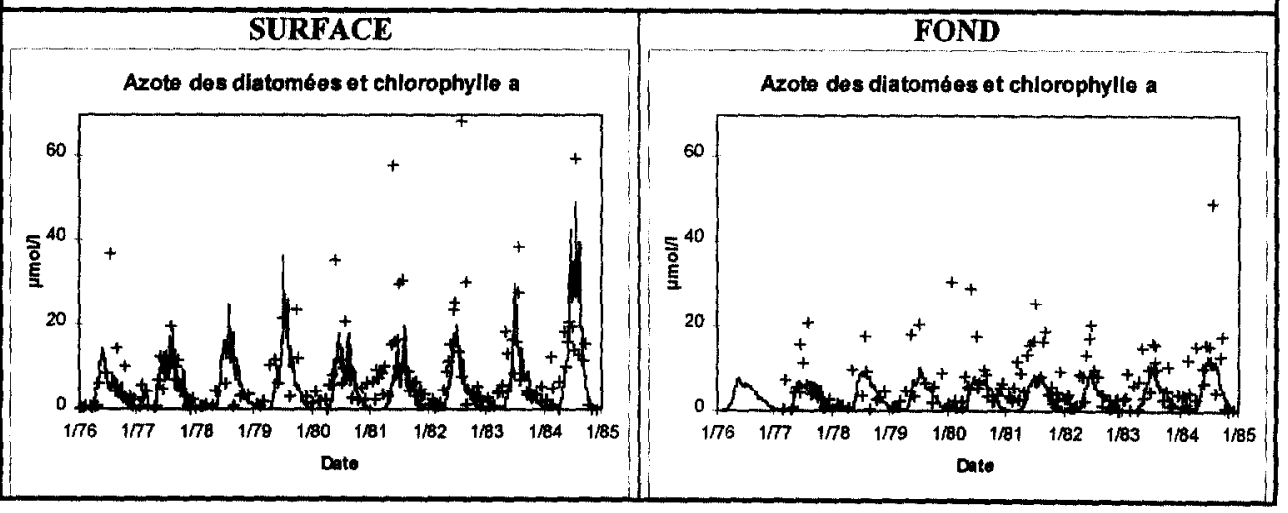

Figure 8. Concentrations mesurées ( + ) et calculées ( - ) en azote minéral dissous (figure 8a), en silicate (figure 8b), en azote des diatomées et en chlorophylle a (figure 8c) au point 2 du RNO (surface et fond), entre 1976 et 1984.

Figure 8. Measured $(+)$ and simulated $(-)$ concentrations of dissolved inorganic nitrogen (figure 8a), silicate (figure 8b), diatom nitrogen and chlorophyll $a$ (figure 8c) at RNO station No. 2, in surface and bottom waters (1976-1984). 


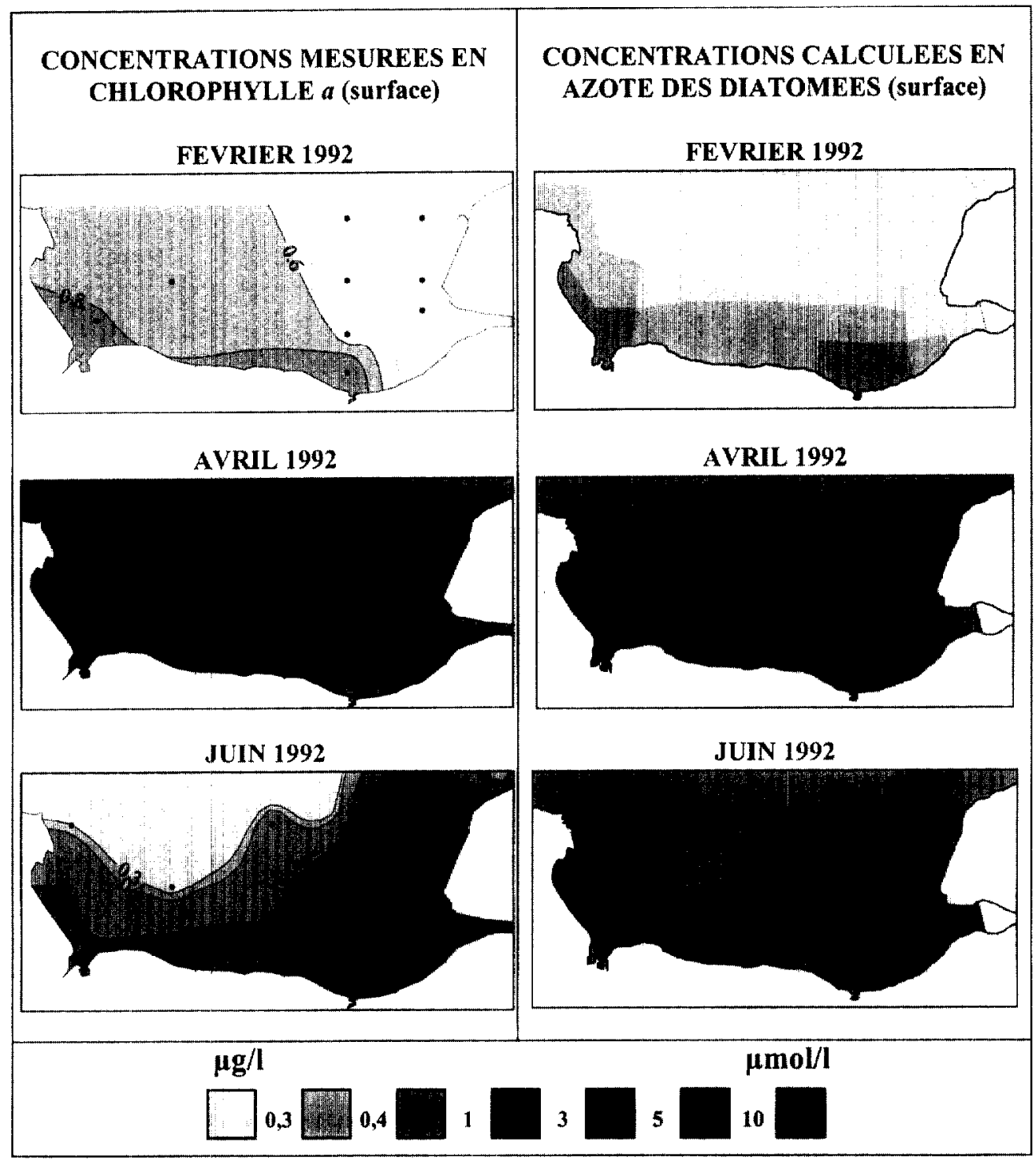

Figure 9. Concentrations mesurées en chlorophylle $a$ et concentrations calculées en azote des diatomées en surface dans la baie de Seine en février, avril et juin 1992.

Figure 9. Measured chlorophyll a concentrations and simulated diatom nitrogen values in surface water of the bay, in February, April and June 1992.

à l'insolation moyenne durant la période de forte production (deuxième trimestre) ; cette valeur moyenne est calculée à partir des insolations journalières utilisées connne variable forçante dans le modèle. La production de diatomées sur la baic implique un recyclage de l'azote phytoplanctonique puisque les apports annuels totaux en azote minéral sur le site sont inféricurs aux valeurs de production ; ces apports, qui varient en effet de 190000 à 300000 t/an (50 000 à 160000 t/an d'origine terrestre, et 140000 t/an d'origine marine), permettent d'estimer à environ $40 \%$ le taux de production régénérée, pourcentage égal à celui calculé par Skogen et al. [42] en mer du Nord. Dans la zone plus réduite du panache de la Seine, la production annuelle de diatonées varie entre 33000 et 


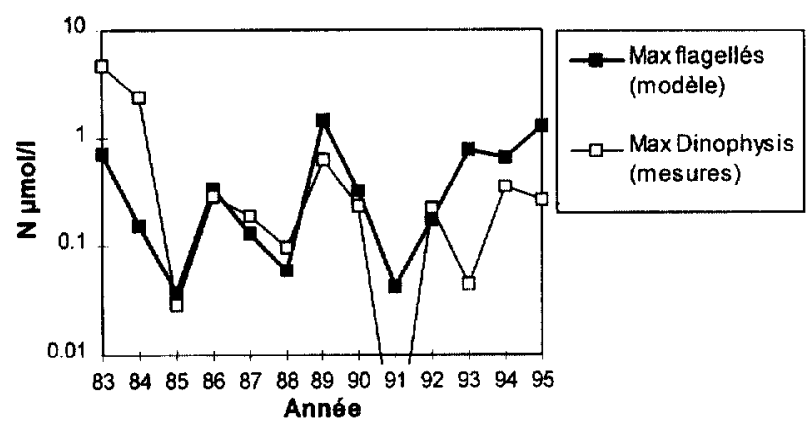

Figure 10. Évolution, de 1983 à 1995, des concentrations maximales en azote des flagellés (calculées par le modèle), el en Dinophysis sp. observées à Antifer (converties en $\mu$ mol L $L^{-1}$ d'azote).

Figure 10. Maximum simulated concentrations of flagellate nitrogen and maximum Dinophysis sp. concentrations (converted into $\mu \mathrm{mol} \mathrm{L}^{-1}$ of nitrogen) observed at Antifer between 1983 and 1995.

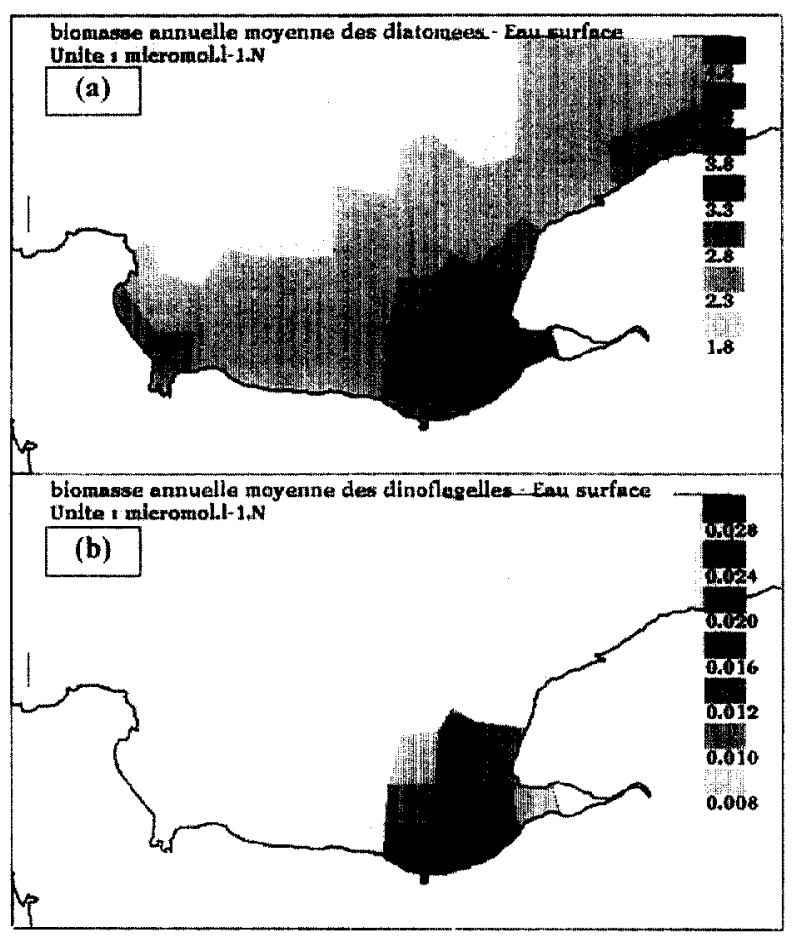

Figure 11. Concentrations moyennes calculées (1976-1995) en azote des diatomées (figure $1 /$ a) et en azote des flagellés (figure $/ / \mathrm{b}$ ), en surface dans la baie de Seine.

Figure 11. Average simulated concentrations of diatom nitrogen (figure 1/a) and flagellate nitrogen (figure $1 / \mathrm{b}$ ) in the surface water of the Bay of Seine, between 1976 and 1995.
56000 t/an, en raison des apports en silicate d'origine terrestre; ce résultat est à rapprocher des observations faites par Videau et al. [49] qui ont mis en évidence le rôle limitant du silicate dans le panache de la Seine durant la période printanière de production des diatomées.

Le modèle a été utilisé, sur les 20 ans considérés (19761995), pour estimer les décalages temporels relatifs à l'apparition des maximums de biomasse phytoplanctonique dans les différents secteurs de la baie. Pour les diatomées, et conformément aux observations, le maximum de hiomasse apparaît d'abord fin avril-début mai dans la bande côtière allant de la baie des Veys au secteur situé au large de l'embouchure de la Seine; le pic de diatomées est ensuite présent sur le reste du site en mai-juin, à l'exception du secteur le plus directement soumis à l'influence de l'estuaire, où le maximum de diatomées est observé en juillet-août. En ce qui concerne les flagellés, le maximum de biomasse apparaît en juillet au large, puis de plus en plus tard lorsque l'on se rapproche de l'estuaire et finalement fin août dans l'embouchure de la Seine, ce qui correspond à la synthèse des observations de Le Grand [27].

Les évolutions pluriannuelles des productions calculées de diatomées et de flagellés dans le panache de la Seine sont représentées sur la figure 12. Pour les diatomées, il n'y a pas d'évolution significative à long terme de leur production, ce qui est cohérent avec la stabilité, sur 20 ans. des apports en silicate par la Seine (figure 3); la même absence d'évolution a été trouvée par Fransz et Verhagen [17] dans la simulation de la production des diatomées sur la côte des Pays-Bas, entre 1930 et 1980.

Pour les flagellés une tendance significative à l'augmentation des productions est mise en évidence par le modèle, malgré la forte variabilité des niveaux annuels. Les facteurs explicatifs de ces variations ont été recherchés par examen de différentes variables, en période de production des flagellés (juillet-août) dans le panache. Le premier facteur explicatif, corrélé positivement à la production calculée des flagellés, est le rapport N/Si du milieu ; la figure 13 représente les valeurs estivales de $\mathrm{N} /$ Si (mesures disponibles à la station 2 du RNO de 1976 à 1984) et les valeurs calculées de la production des flagellés durant la même période. Le deuxième facteur, corrélé positivement à la production des flagellés est la température de l'eau.

Les fortes valeurs du rapport N/Si, durant les années très productives en flagellés reflètent l'épuisement relatif du 


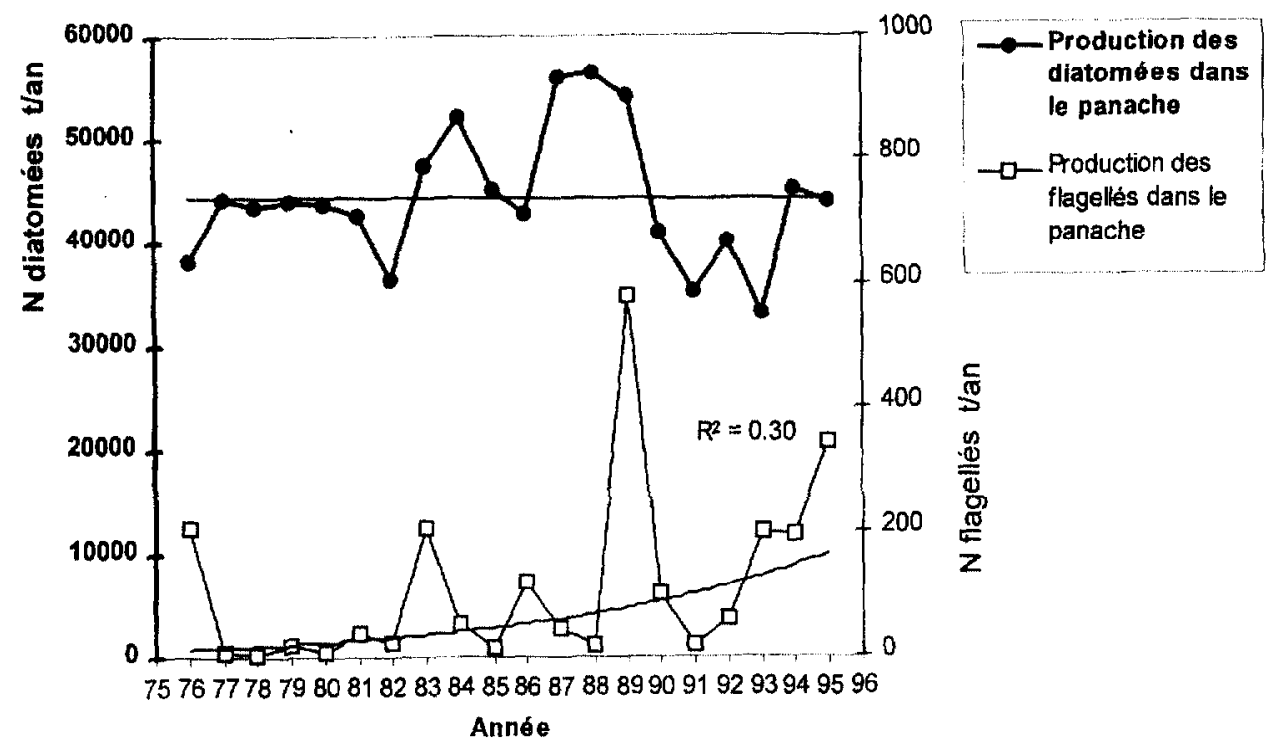

Figure 12. Évolution pluriannuelle des productions calculées de diatomées et de flagellés dans le panache de la Seine.

Figure 12. Time-series of diatom and flagellate simulated productions in the Seine plume.

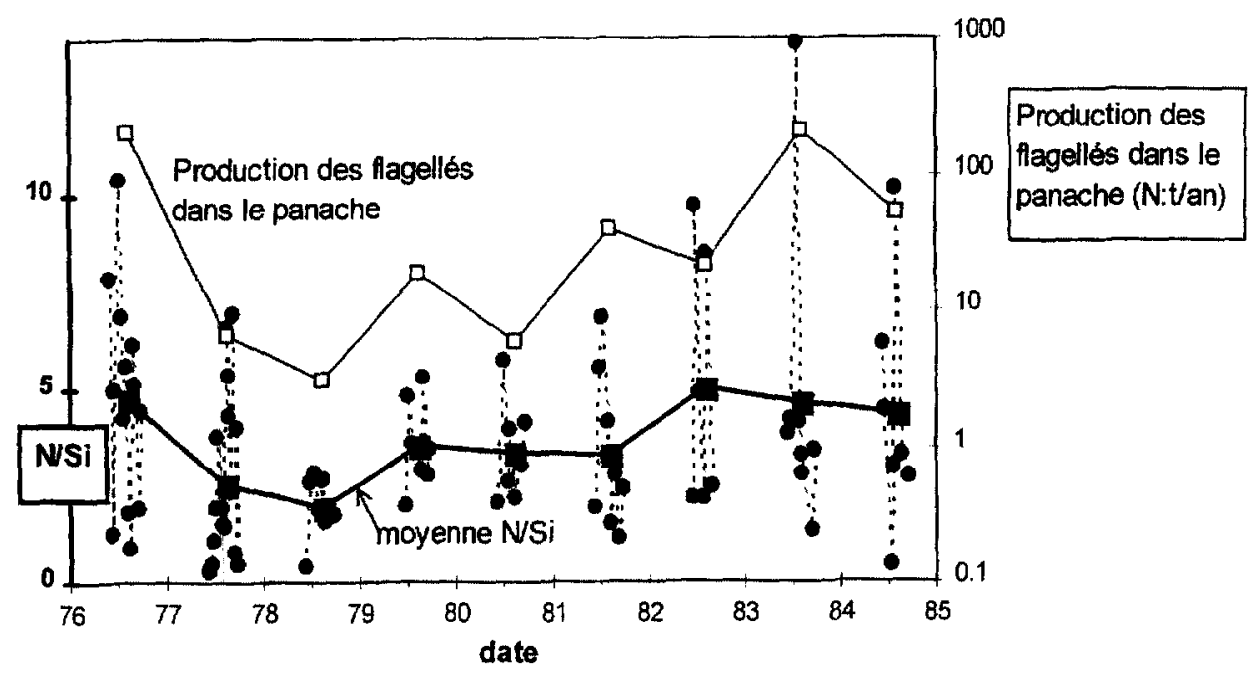

Figure 13. Évolution, en période estivale, du rapport N/Si et de la production des flagellés dans le panache de la Seine.

Figure 13. Time-series of summer N/Si ratio values and flagellate productions in the Seine plume.

milieu en silicate et la disponibilité d'azote pour la croissance des flagellés. Or en juillet-août, le rapport N/Si au cour du panache est étroitement lié à ses valeurs dans les eaux de la Seine $\left(r^{2}=0,73 ; n=20\right)$; il est intéressant de noter que la valeur estivale du rapport $N / S i$ dans les eaux fluviales est fonction du débit; en effet la tendance à l'augmentation des concentrations en azote minéral dissous, et à la diminution des concentrations en silicate lorsque que le débit diminue [31], fait que durant les années d'étiage sévère le rapport N/Si est plus fort que 
lors d'étés pluvieux. Ce même rapport N/Si présente une tendance pluriannuelle à la hausse, indépendante du débit, liée à l'augmentation des teneurs en azote minéral dissous et à la stabilité des teneurs en silicate sur la période 1976-1995 (figure 3). Les observations faites par Radach [37] montrent qu'une évolution similaire du rapport N/Si dans les eaux côtières de la mer du Nord a conduit à l'accroissement des flagellés par rapport aux diatomées entre 1962 et 1984. Les expériences en mésocosmes de Schollhorn et Granéli [41], Escaravage et al. [16] et Sommer [44] mettent en évidence que l'augmentation du rapport N/Si dans le milieu tend à faire augmenter le rapport flagellés/diatomées.

Le modèle utilisé montre que la production estivale des flagellés augmente durant les années où l'étiage est sévère, et lorsque les conditions climatiques sont favorables à l'augmentation des températures de l'eau ; la croissance depuis 1976 des teneurs en azote dans les eaux de la Seine pourrait contribuer à l'accroissement de la production de flagellés.

Afin d'examiner plus précisément l'influence des apports fluviaux de nutriments, en particulier d'azote, sur la production phytoplanctonique dans la baie de Seine, deux simulations ont été faites pour l'année 1995 en réduisant les concentrations fluviales en azote minéral dissous respectivement de 25 et de $50 \%$. Les résultats, rassemblés dans le tableau 11 . montrent que la réduction des flux continentaux d'azote a peu d'influence sur la production des diatomées car celle-ci est essentiellement limitée par le silicate ; leur développement se répartit sur l'ensemble de la baie en bénéficiant des apports marins de nutriments. En revanche la production des flagellés, qui s'effectue majoritairement dans le panache de la Seine, est très sensible aux réductions des flux d'azote.

Enfin le rôle des stratifications sur la production phytoplanctonique a été précisé par une simulation de la

Tableau II. Pourcentages de réduction des productions de diatomées et de flagellés en baie de Seine durant l'année 1995, en fonction des taux de diminution des appons fluviaux d'azote.

Table II. Decreasing rates of diatom and flagellate production related to reductions of nitrogen river loadings.

\begin{tabular}{ccc}
\hline $\begin{array}{c}\text { Pourcentage } \\
\text { de réduction } \\
\text { des apports fluviaux } \\
\text { d'azote }\end{array}$ & \multicolumn{2}{c}{$\begin{array}{c}\text { Pourcentage de réduction } \\
\text { de la production }\end{array}$} \\
\cline { 2 - 3 } & des diatomées & des flagellés \\
\hline $25 \%$ & $1 \%$ & $53 \%$ \\
$50 \%$ & $4 \%$ & $74 \%$ \\
\hline
\end{tabular}

version monocouche du modèle; pour l'année 1995 l'absence de stratification entraîne une légère augmentation de la production globale de diatomées sur la baie $(+14 \%)$, mais les concentrations maximales en diatomées dans le panache sont réduites d'un tiers et apparaissent plus tardivement; cette absence de stratification provoque une forte diminution de la production de flagellés $(-53 \%)$ sur le site.

\section{CONCLUSION}

L'utilisation d'un modèle écologique bicouche sur la baie de Seine, calibré et validé à l'aide des mesures depuis 1976, permet de mieux cerner les facteurs régissant la production phytoplanctonique sur ce site soumis aux apports importants de la Seine.

Les biomasses maximales de phytoplancton se rencontrent dans les zones directement soumises à l'influence des fleuves, en particulier de la Seine. Le modèle montre que la production de diatomées commence à s'établir au printemps, dans la baie des Veys et au large de l'estuaire, puis progresse vers l'embouchure où les plus fortes biomasses sont atteintes en juillet. Les flagellés sont présents d'abord au large, puis atteignent leur biomasse maximale fin août dans l'embouchure. En termes de production, les résultats du modèle sont un peu plus contrastés car la zone du panache estuarien ne contribue qu'à environ $10 \%$ de la production totale des diatomées dans la baie, alors qu'en moyenne près de $40 \%$ des flagellés y sont produits. Ce résultat est à rapprocher des stratifications de la colonne d'eau, par les apports d'eau douce de la Seine, et de leur effet positif sur la production des flagellés.

Les variations pluriannuelles de la production des diatomées dans l'ensemble de la baie apparaissent liées à l'intensité de l'ensoleillement durant la période productive, alors que dans la zone plus réduite du panache, le premier facteur de variation est le silicate apporté au printemps par la Seine ; cela confirmerait le rôle limitant de ce nutriment pour les diatomées dans ce secteur de la baie. La production annuelle des flagellés s'accroît fortement dans le panache de la Seine durant les années où la température estivale des eaux et le rapport $\mathrm{N} / \mathrm{Si}$ sont élevés ; l'augmentation de ce rapport durant la période de production est en relation avec la nature des apports en nutriments par la Seine durant la même période; ainsi lors des années où le débit estival du fleuve est très faible, on observe une chute des apports en silicate plus forte que celle des apports en azote, très probablement à cause de 
la forte réduction du lessivage sur le bassin versant (source essentielle de silicate) et de la forte consommation du silicate par les diatomées fluviales. Les étés secs et chauds seraient donc plus favorables au développement des flagellés dans l'embouchure. La progression depuis 20 ans des apports moyens d'azote par la Seine, parallèlement à la stabilité des apports en silicate, contribue à l'augmentaiion du rapport N/Si dans le milieu ; cette évolution pluriannuelle est à rapprocher de la tendance à l'augmentation des flagellés mise en évidence par le modèle ; celui-ci devrait être amélioré par la formulation explicite du compartiment zooplanctonique et par une meilleure calibration à l'aide des données de terrain récemment acquises, en particulier sur les peuplements de flagellés.

Les résultats obtenus à l'aide du modèle bicouche thermohalin rendent compte des effets de la stratification dans le panache de la Seine et représentent un progrès par rapport au modèle monocouche développé par Ménesguen et al. [31]; néanmoins les accumulations phyto- planctoniques, en particulier de flagellés, observées au sein de la pycnocline en zone côtière [18], ne peuvent être reproduites par ce modèle. La prise en compte de ces processus nécessiterait un modèle tridimensionnel, actuellement en cours de développement sur la baie de Seine. Les connaissances récemment acquises en baie de Seine sur les formes de phosphore liées aux sédiments, sur leur biodisponibilité et sur les processus d'échange particulairedissous [6], permettront d'intégrer cet ćlćment dans la modélisation de la production phytoplanctonique, et d'en améliorer la représentativité.

\section{Remerciements}

Cette étude a été financée par le Programme national d'océanographie côtière (thème eutrophisation). Les auteurs adressent leurs remerciements à Alain Aminot, Roger Kérouel et Philippe Cann pour leur aide à la réalisation de cette publication.

\section{RÉFÉRENCES}

[1] Aminot A., Guillaud J.-F., Andrieux-Loyer F., Kérouel R., Cann P., Apports de nutriments et développement phytoplanctonique en baie de Seine, Oceanol. Acta 21 (6) (1998) 923-935.

[2] Aminot A., Guillaud J.-F., Kérouel R., La baie de Seine : hydrologie, nutriments et chlorophylle (1978-1994), Repère Océan $n^{\circ} 14$, Publication Ifremer (1997) $148 \mathrm{p}$.

[3] Aksnes D.L., Ulvestad K.B., Balino B.M., Bernsten J., Egge J.K., Svendsen E., Ecological modelling in coastal waters: towards predictive physical-chemical-biological simulation models, Ophelia 41 (1995) 5-36.

[4] Andersen V., Modélisation d'écosystèmes pélagiques, Études de processus, thèse doct. univ. P.-et-M.-Curie, Paris (1985) $135 \mathrm{p}$.

[5] Andersen V., Nival P., Modelling of phytoplankton dynamics in an enclosed water column, J. Mar. Biol. Ass. U.K. 69 (1989) 625-646.

[6] Andrieux-Loyer F., Les formes de phosphore particulaire et sédimentaire en environnement côtier, Méthodes d'analyse, biodisponibilité, échange, thèse doct. univ. Bretagne occidentale (1997) $312 \mathrm{p}$.

[7] Bentley D., Maillard-Quisthoudt C., Caractéristiques physicochimiques de profils côte-large en Manche centrale, J. Rech. Océanogr 15 (1990) 135-141.

[8] Cellule Antipollution de la Seine, Étude des pollutions en Seine, Ministère de l'environnement, Service maritime de la
Seine Maritime ( $3^{\mathrm{e}}$ Section), documents non paginés (1976 à 1995).

[9] Chapelle A., Modélisation d'un écosystème marin côtier soumis à l'eutrophisation : la baie de Vilaine, étude du phytoplancton et du bilan en oxygène, thèse doct. univ. Paris-VI (1991) $201 \mathrm{p}$.

[10] Cnexo, Centre National pour l'Expoitation des océans, Campagnes Écomanche 1-II, État de référence écologique de la Manche, Résultats des campagnes à la mer, $n^{\circ} 21-1$, Cnexo (éd.) (1980) $60 \mathrm{p}$.

[11] Conley D.J., Malone T.C., Annual cycle of dissolved silicate in Chesapeake Bay: implications for the production and fate of phytoplankton biomass, Mar. Ecol. Prog. Ser. 81 (1992) 121128.

112] Duplessix P., Bilan de la présence de Dinophysis en Normandie de 1994 à 1996, Rapport Ifremer-DEL/Port-en-Bessin (1996) $61 \mathrm{p}$.

[13] Droop M.R., Some thoughts on nutrient limitation in algae, J. Phycol. 9 (1973) 264-272.

[14] Eppley R.W., Rogers J.N., McCarthy J.J., Half-saturation constants for uptake of nitrate and ammmonium by marine phytoplankton, Limnol. Oceanogr. 14 (1969) 912-920.

[15] Eppley R.W., Temperature and phytoplankton growth in the sea, Fish. Bull. 70 (1972) 1063-1085. 
[16] Escaravage V., Prins T.C., Nijdam C., Smaal A.C., Peeters J.C.H., The response of phytoplankton communities to Ninput reduction results from mesocosm experiments, ICES Annual Science Conference; C.M. 1995/T:10 (1995) 16 p.

[17] Fransz H.G., Verhagen J.H.G., Modeling research on the production cycle of phytoplankton in the southern Bight of the North Sea in relation to riverborne nutrient loads, Neth. J. Sea Res. 19 (1985) 241-250.

[18] Gentien P., Lunven M., Lehaître M., Duvent J.L., in situ depth profiling of particle sizes, Deep-Sea Res. 42 (8) (1995) 12971312.

[19] Harrison P.J., Conway H.L., Holmes R.W., Davis C.O., Marine diatoms growth in chemostats under silicate or ammonium limitation III, Cellular chemical composition and morphology of Chaetoceros debilis, Skeletonema costatum and Thalassiosira gravida, Mar. Biol. 43 (1997) 19-31.

[20] Hoch T., Modélisation du cycle biogéochimique des éléments limitant la production biologique en Manche (N, P, Si), thèse univ. Paris-VII (1995) 205 p.

[21] Huntley M.E., Yellow water in La Jolla Bay, California, July 1980 II, Suppression of zooplankton grazing. J. Exp. Mar. Biol. Ecol. 63 (1982) 81-91.

[22] I.O.S. Institute of Oceanographic Sciences, Geochemical studies in the English Channel, Cruise report No 192, Natural Environment Research Council (êd.) (1987) $40 \mathrm{p}$.

[23] Jonge V.N. de, Boynton W., D'Elia C.F., Elmgren R., Welsh B.L.. Responses to developments in eutrophication in four different North Atlantic estuarine systems, in : Dyer K.R., Orth R.J. (éd.), Changes in Fluxes in estuaries: implications from Science to Management, Olsen \& Olsen, Fredensborg, 1994 , 179-196.

[24] Justic D., Long-term eutrophication of the northern Adriatic Sea, Mar. Pollut. Bull. 18 (1991) 281-284.

[25] Lassus P., Proniewski F., Maggi P., Truquet P., Bardouil M., Wind-induced toxic blooms of Dinophysis cf. acuminata in the Antifer area (France), in : Smayda T.J., Shimizı Y. (éd.), Toxic Phytoplancton Blooms in the Sea, Elsevier Science Publishers B.V., 1993, 519-523.

[26] Le Fèvre-Lehoërff G., Erard-Le Denn E., Arzul G., Planktonic ecosystems in the Channel, Trophic relations, Oceanol. Acta. 16 (1993) 66l-670.

[27] Le Grand J., Bilan du réseau de surveillance phytoplanctonique en Normandie (1989-1992), Ifremer, Rapport interne DEL / 94.09 / Port-en Bessin (1994) 72 p.

[28] Le Pape O., Modélisation des cycles biogéochimiques des éléments limitant la production phytoplanctonique en Rade de Brest, thèse doct. Fcole nat. sup. agron. Rennes (1996) $195 \mathrm{p}$.

[29] Maggi P., Truquet P., Mornet F., Lassus P., Distribution verticale, à basse et pleine mers, du phytoplancton estival en baie de Seine, Ifremer, Rapp. int. DEL / 92.06 / Nantes (1992) 22 p.

[30] Meksumpun S., Montani S., Ichimi K., Tada K., Yoshimatsu S., Okaichi T., Relationships between the biochimical composition and the environmental conditions of Gymnodinium sp. red tide in the Seto inland sea, in: Lassus P., Arzul G., Erard
E., Gentien P., Marcaillou C. (Eds.), Harmful Marine Algal Blooms, Techn. Doc. Lavoisier, 1995, 621-626.

[31] Ménesguen A., Guillaud J-F., Aminot A., Hoch T., Modelling the eutrophication in a river plume: the Seine river case study (France), Ophelia 42 (1995) 205-225

[32] Ménesguen A., Hoch T., Modelling the biogeochemical cycles of elements limiting primary production in the English Channel, I. Role of thermohaline stratification, Mar. Ecol. Prog. Ser. 146 (1997) 173-188.

[33] Mortain-Bertrand A., Descolas-Gros C., Jupin H., Growth, photosynthesis and carbon metabolism in the temperate marine diatom Skeletonema costatum adapted to low temperature and low photon-flux density, Mar. Biol. 100 (1988) 135141 .

[34] Niiler P.P., Kraus E.B., One-dimensional models of the upper ocean, in: Kraus B.B., (éd.), Modelling and prediction of the upper layer of the ocean, Proceedings of a NATO Advanced Study Institute. Pergamon Press, Oxford, 1977, 145-172.

[35] Paasche E., Silicon and the ecology of marine plankton diatoms II Silicate-uptake kinetics in five diatom species, Mar. Biol. 19 (1973) 262-269.

[36] Peperzak L., Snoeijer G.J., Dijkema R., Gieskes W.W.C., Joordens J., Peeters J.C.H., Schol C., Vrieling E.G., Zevenboom W. Development of a Dinophysis acuminata bloom in the river Rhine plume (North Sea), in : Yasumoto T., Oshima Y., Fukuyo Y. (éd.), Harmful and Toxic Algal Blooms, Intergovernmental Oceanographic Commission of Unesco, 1996, 273-276.

[37] Radach G., Ecosystem functioning in the German Bight under continental nutrient inputs by rivers, Estuaries 15 (1992) 477 496.

[38] RNO Réseau national d'observation de la qualité du milieu marin, Recueil des observations, Campagnes 1976 à 1984 , Ministère de l'environnement - Cnexo (1976-1984).

[39] RNO Réseau national d'observation de la qualité du milieu marin, Surveillance des éléments nutritifs et de la chlorophylle, in: Travaux du RNO, Surveillance du milieu marin: édition 1992-1993, Ministère de l'environnement - Ifremer 9-26 (1993).

[40] Riley G.A., Transparency-chlorophyll relations, Limnol. Oceanogr. 20 (1975) 150-152.

[41] Schollhorn E., Graneli E., Is the increase of flagellates in coastal waters caused by changes in ratios of $\mathrm{N}, \mathrm{P}$ and $\mathrm{Si}$ ? in : Smayda T.J., Shimizu Y. (éd.), Toxic Phytoplancton Bloorns in the Sea, Elsevier Science Publishers B.V., 1993, 811-817.

[42] Skugen M.D., Svendsen E., Berntsen J., Aksnes D., Ulvestad K.B., Modelling of primary production in the North Sea using a coupled three-dimensional physical-chemical-hiological model, Estuar. Coast. Shelf Sci. 41 (1995) 545-565.

[43] Smayda T.J., Novel and nuisance phytoplankton blooms in the sea: evidence for global epidemic, in : Graneli E., Sundstrom B., Edler R., Anderson D.M., (éd.), Toxic marine phytoplankton, Elsevier, New York, 1990, 29-40. 
[44] Sommer U., Eutrophication related changes in phytoplankton species composition: is there a role of nutrient competition ? ICES Annual Science Conference; C.M. 1995/T:7 (1995) 6 p.

[45] Soulsby R.L., Hamm L., Klopman G., Myrhaug D., Simons R.R., Thomas G.P., Wave-current interaction within and outside the bottom boundary layer, Coast. Engin. 21 (1993) 4169.

[46] Tappin A.D., Studies of trace metals in shelf waters of the British Isles, thesis, univ. Southampton, (1988) $332 \mathrm{p}$.
[47] Titman D., Kilham P., Sinking in freshwater phytoplankton: some ecological implications of cell nutrient status and physical mixing processes. Limnol. Oceanogr. 21 (1976) 409-417.

[48] Turner R.E., Rabalais N.N., Changes in Mississippi River water quality this century - implications for coastal food webs. BioScience 41 (1991) 140-147.

1491 Videau C., Ryckaert M., L'Helguen S., Phytoplancton en baie de Seine, Influence du panache fluvial sur la production primaire, Oceanol. Acta 21 (6) (1998) 907-921. 